\title{
Milagro Limits and HAWC Sensitivity for the Rate-Density of Evaporating Primordial Black Holes
}

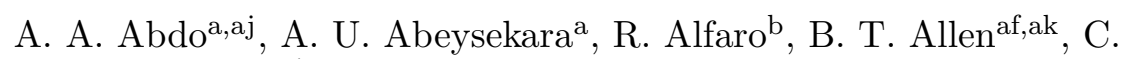
Alvarez $^{\text {c }}$,J. D. Álvarez ${ }^{\mathrm{d}}$, R. Arceo ${ }^{\mathrm{c}}$, J. C. Arteaga-Velázquez $^{\mathrm{d}}, \mathrm{T}$. Aune $^{\text {aa,al }}$, H. A. Ayala Solares ${ }^{\mathrm{e}}$, A. S. Barber ${ }^{\mathrm{f}}$, B. M. Baughman ${ }^{\mathrm{g}}$, N. Bautista-Elivar $^{\mathrm{h}}$, J. Becerra Gonzalez ${ }^{\mathrm{i}, \mathrm{g}}$, E. Belmont ${ }^{\mathrm{b}}$, S. Y. BenZvi ${ }^{\text {ae }}$, D. Berleyg $^{\mathrm{g}}$, M. Bonilla Rosales ${ }^{\mathrm{k}}$, J. Braun ${ }^{\mathrm{g}}$, R. A. Caballero-Lopez ${ }^{\mathrm{l}}$, K. S. Caballero-Mora $^{\mathrm{m}}$, A. Carramiñana ${ }^{\mathrm{k}}$, M. Castillon $^{\mathrm{n}}$, G. E. Christopher ${ }^{\mathrm{ag}}$, U. Cotti $^{\mathrm{d}}$, J. Cotzomi ${ }^{\mathrm{n}}$, E. de la Fuente ${ }^{\mathrm{o}}$, C. De León ${ }^{\mathrm{d}}$, T. DeYounga,p, R.

Diaz Hernandez $^{k}$, L. Diaz-Cruz ${ }^{n}$, J. C. Díaz-Vélez ${ }^{j}$, B. L. Dingus ${ }^{q}$, M. A. DuVernois ${ }^{j}$, R. W. Ellsworth ${ }^{\mathrm{r}, \mathrm{g}}$, D. W. Fiorino ${ }^{\mathrm{j}}$, N. Fraija ${ }^{\mathrm{s}}$, A. Galindo ${ }^{\mathrm{k}}$, F. Garfias $^{\mathrm{s}}$, M. M. González ${ }^{\mathrm{s}}$, J. A. Goodman ${ }^{\mathrm{g}}$, V. Grabski ${ }^{\mathrm{b}}$, M. Gussert ${ }^{\mathrm{t}}$, Z. Hampel-Arias ${ }^{j}$, J. P. Harding ${ }^{\mathrm{q}}$, E. Hays ${ }^{\mathrm{i}}$, C. M. Hoffman ${ }^{\mathrm{q}}$, C. M. Hui ${ }^{\mathrm{e}}$, P. Hüntemeyer ${ }^{\mathrm{e}}$, A. Imran ${ }^{\mathrm{j}}$, A. Iriarte ${ }^{\mathrm{s}}$, P. Karn ${ }^{\mathrm{j}}$, D. Kieda ${ }^{\mathrm{f}}$, B. E.

Kolterman $^{\text {ag }}$, G. J. Kunde ${ }^{\mathrm{q}}$, A. Laral, R. J. Lauer ${ }^{\mathrm{u}}$, W. H. Lee ${ }^{\mathrm{s}}$, D.

Lennarz $^{\mathrm{v}}$, H. León Vargas ${ }^{\mathrm{b}}$, E. C. Linares ${ }^{\mathrm{d}}$, J. T. Linnemann ${ }^{\mathrm{a}}$, M. Longo ${ }^{\mathrm{t}}$, R. Luna-GarcIa ${ }^{\mathrm{w}}$, J. H. MacGibbon ${ }^{\text {ad }}$, A. Marinelli ${ }^{\mathrm{b}}$, S. S. Marinelli ${ }^{\mathrm{a}}$, H.

Martinez $^{\mathrm{m}}$, O. Martinez ${ }^{\mathrm{n}}$, J. Martínez-Castro ${ }^{\mathrm{w}}$, J. A. J. Matthews ${ }^{\mathrm{u}}$, J.

McEnery ${ }^{\mathrm{i}}$, E. Mendoza Torres ${ }^{\mathrm{k}}$, A. I. Mincer ${ }^{\mathrm{ag}}$, P. Miranda-Romagnolix ${ }^{\mathrm{x}}$, E.

Morenon $^{n}$, T. Morgan ${ }^{\text {ah }}$, M. Mostafáp ${ }^{2}$ L. Nellen ${ }^{\mathrm{y}}$, P. Nemethy ${ }^{\text {ag }}$, M.

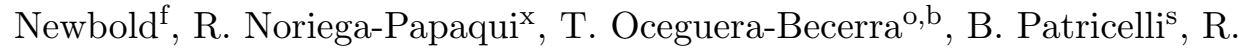
Pelayo $^{w}$, E. G. Pérez-Pérez ${ }^{\mathrm{h}}$, J. Pretz ${ }^{\mathrm{p}}$, C. Rivière ${ }^{\mathrm{g}, \mathrm{s}}$, D. Rosa-González ${ }^{\mathrm{k}}$, E. Ruiz-Velasco ${ }^{\mathrm{b}}$, J. Ryan ${ }^{\mathrm{z}}$, H. Salazar ${ }^{\mathrm{n}}$, F. Salesa ${ }^{\mathrm{p}}$, A. Sandoval ${ }^{\mathrm{b}}$, P. M. Saz Parkinson ${ }^{\text {aa,ai }}$, M. Schneider ${ }^{\text {aa }}$, S. Silich ${ }^{\mathrm{k}}$, G. Sinnis ${ }^{\mathrm{q}}$, A. J. Smith ${ }^{\mathrm{g}}$, D.

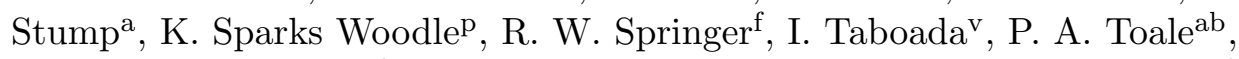
K. Tollefson ${ }^{\mathrm{a}}$, I. Torres ${ }^{\mathrm{k}}$, T. N. Ukwatta ${ }^{\mathrm{a}, \mathrm{q}}$, V. Vasileiou $^{\mathrm{g}, \mathrm{am}}$, L. Villaseñor ${ }^{\mathrm{d}}$, T. Weisgarber ${ }^{j}$, S. Westerhoff ${ }^{j}$, D. A. Williams ${ }^{\text {aa }}$, I. G. Wisher ${ }^{j}$, J. Wood $^{g}$,

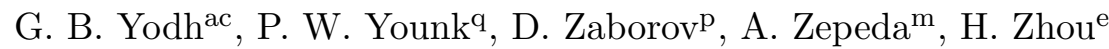

\footnotetext{
${ }^{a}$ Department of Physics and Astronomy, Michigan State University, East Lansing, MI, $U S A$

${ }^{b}$ Instituto de Física, Universidad Nacional Autónoma de México, Mexico D.F., Mexico

${ }^{c}$ CEFyMAP, Universidad Autónoma de Chiapas, Tuxtla Gutiérrez, Chiapas, México

${ }^{d}$ Universidad Michoacana de San Nicolás de Hidalgo, Morelia, Mexico

${ }^{e}$ Department of Physics, Michigan Technological University, Houghton, MI, USA

${ }^{f}$ Department of Physics and Astronomy, University of Utah, Salt Lake City, UT, USA

${ }^{g}$ Department of Physics, University of Maryland, College Park, MD, USA

${ }^{h}$ Universidad Politecnica de Pachuca, Pachuca, Hgo, Mexico

${ }^{i}$ NASA Goddard Space Flight Center, Greenbelt, MD 207r1, USA

${ }^{j}$ Department of Physics, University of Wisconsin-Madison, Madison, WI, USA
} 
${ }^{k}$ Instituto Nacional de Astrofísica, Óptica y Electrónica, Tonantzintla, Puebla, México ${ }^{l}$ Instituto de Geofísica, Universidad Nacional Autónoma de México, Mexico D.F., Mexico

${ }^{m}$ Physics Department, Centro de Investigacion y de Estudios Avanzados del IPN, Mexico City, DF, Mexico

${ }^{n}$ Facultad de Ciencias Físico Matemáticas, Benemérita Universidad Autónoma de Puebla, Puebla, Mexico

${ }^{o}$ IAM-Dpto. de Fisica; Dpto. de Electronica (CUCEI), IT.Phd (CUCEA), Phys_Mat. Phd (CUVALLES), Universidad de Guadalajara, Jalisco, Mexico

${ }^{p}$ Department of Physics, Pennsylvania State University, University Park, PA, USA

${ }^{q}$ Physics Division, Los Alamos National Laboratory, Los Alamos, NM, USA

${ }^{r}$ School of Physics, Astronomy, and Computational Sciences, George Mason University, Fairfax, VA, USA

${ }^{s}$ Instituto de Astronomía, Universidad Nacional Autónoma de México, Mexico D.F., Mexico

${ }^{t}$ Colorado State University, Physics Dept., Ft Collins, CO 80523, USA

${ }^{u}$ Dept of Physics and Astronomy, University of New Mexico, Albuquerque, NM, USA

${ }^{v}$ School of Physics and Center for Relativistic Astrophysics - Georgia Institute of Technology, Atlanta, GA, USA 30332

${ }^{w}$ Centro de Investigacion en Computacion, Instituto Politecnico Nacional, Mexico City, Mexico

${ }^{x}$ Universidad Autónoma del Estado de Hidalgo, Pachuca, Hidalgo, Mexico

${ }^{y}$ Instituto de Ciencias Nucleares, Universidad Nacional Autónoma de México, Mexico D.F., Mexico

${ }^{z}$ Space Science Center, University of New Hampshire, Durham, NH, USA

${ }^{a}$ Santa Cruz Institute for Particle Physics, University of California, Santa Cruz, Santa Cruz, CA, USA

${ }^{a b}$ Department of Physics \& Astronomy, University of Alabama, Tuscaloosa, AL, USA

${ }^{a c}$ Department of Physics and Astronomy, University of California, Irvine, Irvine, CA, $U S A$

${ }^{a d}$ Department of Physics, University of North Florida, Jacksonville, FL 32224, USA.

${ }^{a e}$ Department of Physics and Astronomy, University of Rochester, Rochester, NY, USA.

${ }^{a f}$ Department of Physics and Astronomy, University of California, Irvine, CA 92697

${ }^{a g}$ Department of Physics, New York University, 4 Washington Place, New York, NY 10003

${ }^{a h}$ Department of Physics, University of New Hampshire, Morse Hall, Durham, NH 03824

${ }^{a i}$ Department of Physics, The University of Hong Kong, Pokfulam Road, Hong Kong, China

${ }^{a j}$ Current address: Operational Evaluation Division, Institute for Defense Analyses, 4850 Mark Center Drive, Alexandria, VA 22311-1882

${ }^{a k}$ Current address: Harvard-Smithsonian Center for Astrophysics, Cambridge, MA 02138

${ }^{a l}$ Current address: Department of Physics and Astronomy, University of California, Los Angeles, CA 90095

${ }^{a m}$ Current address: Laboratoire Univers et Particules de Montpellier, Université Montpellier 2, CNRS/IN2P3, CC 72, Place Eugène Bataillon, F-34095 Montpellier Cedex 5, France 


\begin{abstract}
Primordial Black Holes (PBHs) are gravitationally collapsed objects that may have been created by density fluctuations in the early universe and could have arbitrarily small masses down to the Planck scale. Hawking showed that due to quantum effects, a black hole has a temperature inversely proportional to its mass and will emit all species of fundamental particles thermally. PBHs with initial masses of $\sim 5.0 \times 10^{14} \mathrm{~g}$ should be expiring in the present epoch with bursts of high-energy particles, including gamma radiation in the $\mathrm{GeV}-\mathrm{TeV}$ energy range. The Milagro high energy observatory, which operated from 2000 to 2008, is sensitive to the high end of the PBH evaporation gamma-ray spectrum. Due to its large field-of-view, more than $90 \%$ duty cycle and sensitivity up to $100 \mathrm{TeV}$ gamma rays, the Milagro observatory is well suited to perform a search for PBH bursts. Based on a search on the Milagro data, we report new $\mathrm{PBH}$ burst rate density upper limits over a range of PBH observation times. In addition, we report the sensitivity of the Milagro successor, the High Altitude Water Cherenkov (HAWC) observatory, to $\mathrm{PBH}$ evaporation events.
\end{abstract}

Keywords: Primordial Black Holes

\title{
1. Introduction
}

Primordial Black Holes (PBHs) are created from density inhomogeneities in many scenarios of the early universe [1]. The initial mass of a $\mathrm{PBH}$ is expected to be roughly equal to or smaller than the horizon or Hubble mass at formation, giving possible PBH masses ranging from that of supermassive black holes down to the Planck mass. PBH production can thus have observable consequences today spanning from the largest scales, for example influencing the development of large-scale structure in the Universe, to the smallest scales, for example enhancing local dark matter clustering. Additionally, $\mathrm{PBHs}$ in certain mass ranges may constitute a fraction of the dark matter in the universe [1]. For particle physics, the greatest interest is in the radiation directly emitted by a black hole. By evolving an ingoing solution past a gravitationally collapsing object, Hawking showed that a black hole will thermally emit ('evaporate') all available species of fundamental particles [2] with a temperature inversely proportional to the black hole mass.

*Corresponding author: T. N. Ukwatta (tilan.ukwatta@gmail.com) 
PBHs with an initial mass of $\sim 5.0 \times 10^{14} \mathrm{~g}$ should be expiring now with bursts of high-energy particles, including gamma radiation in the $\mathrm{GeV}-$ TeV energy range [3].

Detection of radiation from a $\mathrm{PBH}$ burst would provide valuable insights into the early universe and many areas of physics, as well as confirm the amalgamation of classical thermodynamics with general relativity. Observed PBH radiation will give access to particle physics at energies higher than those which will likely ever be achievable in terrestrial accelerators. Non-detection of PBHs in dedicated searches also gives important information. One of the most important cosmological motivations for $\mathrm{PBH}$ searches is to place limits on the initial density fluctuation spectrum of the early universe. In particular, PBHs can form from the quantum fluctuations associated with many types of inflationary scenarios [4]. Other PBH formation mechanisms include those associated with cosmological phase transitions, topological defects or an epoch of low pressure (soft equation of state) in the early universe (for a review see [1]).

Evaporating PBHs are candidate sources for gamma-ray telescopes, as they produce short bursts of gamma rays. While most gamma-ray bursts (GRBs) are generally thought to be produced by the collapse of massive stars (long duration GRBs) or the merger of compact objects (short duration GRBs) at cosmological distances [5], some short duration GRBs show behavior, such as large offsets from the host galaxy or anisotropic sky distribution, that may indicate a more local origin. Models of PBH evaporation based on Standard Model physics predict a unique $\mathrm{TeV}$ gamma-ray spectrum [6].

Various detectors have searched for PBH events using direct and indirect methods. These methods probe the $\mathrm{PBH}$ distribution on various distance scales. One can probe the $\mathrm{PBH}$ density on the cosmological scale using the $100 \mathrm{MeV}$ extragalactic gamma-ray background, which produces a limit on the corresponding cosmological average $\mathrm{PBH}$ burst rate density of $<10^{-6} \mathrm{pc}^{-3} \mathrm{yr}^{-1}[1 ; 7]$. On the galactic scale, if PBHs are clustered in the Galaxy, we would expect to see an enhancement in the local PBH density and anisotropy in the $100 \mathrm{MeV}$ gamma-ray measurements. Indeed, such an anisotropy has been detected and results in a corresponding Galactic PBH burst limit of $<0.42 \mathrm{pc}^{-3} \mathrm{yr}^{-1}$ [8]. On the kiloparsec scale, the Galactic antiproton background can be used to give a PBH burst limit of $<$ $0.0012 \mathrm{pc}^{-3} \mathrm{yr}^{-1}[9]$. However, the antiproton-derived limit depends on the assumed PBH distribution within the Galaxy and the propagation of antiprotons through the Galaxy, as well as the production and propagation of the secondary antiproton component produced by interactions of cosmic-ray 
nuclei with the interstellar gas. On the parsec scale, the $\mathrm{PBH}$ burst limits are directly set by searches for the detection of individual bursting $\mathrm{PBH}$ and are independent of assumptions of PBH clustering. The best direct search limits come from the Very High Energy (VHE) searches conducted with Imaging Air Cherenkov Telescopes (IACTs) and Extensive Air Shower (EAS) arrays. On the parsec scale, the current best PBH limit from direct searches is $<1.4 \times 10^{4} \mathrm{pc}^{-3} \mathrm{yr}^{-1}[10]$.

\section{Milagro and HAWC Observatories}

In this paper, we present new $\mathrm{PBH}$ burst limits based on the direct search method using the data from the Milagro observatory. These limits are obtained assuming the standard model of Hawking radiation and particle physics $[11 ; 6]$. Milagro was a water Cherenkov gamma-ray observatory (EAS type) sensitive to gamma rays in the energy range $\sim 50 \mathrm{GeV}$ to 100 $\mathrm{TeV}$. The observatory was located near Los Alamos, New Mexico, USA at latitude $35.9^{\circ}$ north, longitude $106.7^{\circ}$ west and an altitude of $2630 \mathrm{~m}$, and was operational from 2000 to 2008 [12]. The Milagro detector had two components: a central rectangular $60 \mathrm{~m} \times 80 \mathrm{~m} \times 7 \mathrm{~m}$ reservoir filled with purified water and an array of 175 smaller outrigger (OR) tanks distributed over an area of $200 \mathrm{~m} \times 200 \mathrm{~m}$ surrounding the reservoir. The reservoir was light-tight and instrumented with two layers of 8-inch photomultiplier tubes (PMTs). The top layer consisted of 450 PMTs (the 'air-shower' layer) 1.5 $\mathrm{m}$ below the water surface and the bottom layer had 273 PMTs (the 'muon' layer) $6 \mathrm{~m}$ below the surface. Each outrigger tank contained one PMT. The observatory detected VHE gamma rays by detecting the Cherenkov light generated by the secondary particles from the gamma-ray-induced air shower as the secondary particles passed through the water. Various components of the detector were used to measure the direction of the gamma ray and to reduce the background due to hadron-induced showers. Because of its large field-of-view, $\sim 2 \mathrm{sr}$, and high duty cycle, over $90 \%$, Milagro was well suited to search for burst emission from PBHs.

In this paper we also present the sensitivity of the High Altitude Water Cherenkov (HAWC) observatory to PBH bursts. HAWC, the successor to Milagro, is the next generation VHE observatory presently under construction at Sierra Negra, Mexico at an altitude of 4100m. HAWC will consist of 300 water tanks, each $7.3 \mathrm{~m}$ in diameter and $4.5 \mathrm{~m}$ deep. Each tank will house three 8-inch PMTs (reused from Milagro) and one 10-inch PMT [13]. The PMTs will detect Cherenkov light from secondary particles created in extensive air showers induced by VHE gamma rays of energy in the range 
$\sim 50 \mathrm{GeV}$ to $100 \mathrm{TeV}$. HAWC has two data acquisition (DAQ) systems: the main DAQ and the scaler DAQ. The main DAQ system measures the arrival direction and energy of the high-energy gamma-rays by timing the arrival of particles on the ground. The direction of the original primary particle may be resolvable with an error of between 0.1 and 2.0 degrees depending on its energy and location in the sky. The scaler DAQ system counts the number of hits in each PMT, allowing a search for excesses over background noise. The scaler DAQ system is more sensitive to lower energy air showers than the main DAQ system. HAWC has a large field-of-view (1.8 sr corresponding to $1 / 7$ th of the sky) and will have a high duty cycle of greater than $90 \%$. Thus, HAWC will be able to observe high-energy emission from gamma-ray transients [14].

\section{Methodology}

\subsection{Primordial Black Hole Burst Spectrum}

The properties of the final burst of radiation from a $\mathrm{PBH}$ depend on the physics governing the production and decay of high-energy particles. As the black hole evaporates, it loses mass and hence its temperature and the number of particle species that it emits increase until the end of its evaporation lifetime. In the Standard Evaporation Model (SEM) [11; 15], a $\mathrm{PBH}$ should directly radiate those fundamental particles whose Compton wavelengths are on the order of the size of the black hole. When the black hole temperature exceeds the Quantum Chromodynamics (QCD) confinement scale (250-300 MeV), quarks and gluons should be directly emitted by the black hole $[7 ; 11]$. The quarks and gluons will then fragment and hadronize as they stream away from the black hole, analogous to the jets seen in terrestrial accelerators $[11 ; 3]$. On astrophysical timescales, the jet products will decay into photons, neutrinos, electrons, positrons, protons and anti-protons.

Detailed studies using the SEM to simulate the particle spectra from black holes with temperatures of $1-100 \mathrm{GeV}$ have shown that the gammaray spectrum is dominated by the photons produced by neutral pion decay in the Hawking-radiated QCD jets and is broadly peaked at photon energies of $\sim 100 \mathrm{MeV}$. The photons which are directly radiated (not the result of decay of other primary particles) are visible as a much smaller peak at a much higher photon energy proportional to the black hole temperature [11]. As the evaporation proceeds to higher temperatures, the greater will be the number of emitted fundamental particle degrees of freedom. As new particle degrees of freedom become available, the luminosity of the burst 
will increase. The energy spectrum is determined by the correct high energy particle physics model. In this work, we will assume the SEM as our emission and particle physics model.

For temperatures well below the Planck temperature, the temperature $(T)$ of a black hole can be expressed in terms of the remaining evaporation lifetime $(\tau)$ of the black hole (that is, the time left until the black hole stops evaporating) as follows $[15 ; 16]$ :

$$
T \simeq\left[4.8 \times 10^{11}\left(\frac{1 \mathrm{sec}}{\tau}\right)\right]^{1 / 3} \mathrm{GeV} .
$$

The emission rate increases as the black hole shrinks and therefore the remaining evaporation lifetime decreases as $T$ increases. For black holes with temperatures greater than several $\mathrm{GeV}$ at the start of the observation, the time integrated photon flux can be parameterized as [16]

$$
\frac{d N}{d E} \approx 9 \times 10^{35} \text { particles } \mathrm{m}^{-2} \mathrm{GeV}^{-1}\left\{\begin{array}{l}
\left(\frac{1 G e V}{T}\right)^{3 / 2}\left(\frac{1 G e V}{E}\right)^{3 / 2}, \quad E<T \\
\left(\frac{1 G e V}{E}\right)^{3}, \quad E \geq T
\end{array}\right.
$$

for gamma ray energies $E \gtrsim 10 \mathrm{GeV}$. The $E^{-3}$ fall off at $E \geq T$ comes from the $\tau \propto T^{-3}$ dependence in Equation 1, which is less steep than the high energy exponential tail in the instantaneous Hawking spectrum at each $T$ [16]. Figure 1 shows the resulting gamma-ray spectrum for various $\mathrm{PBH}$ remaining lifetimes ranging from $0.001 \mathrm{~s}$ to $100 \mathrm{~s}$.

\subsection{Detectable Volume Estimation}

In order to calculate the upper limits on the $\mathrm{PBH}$ burst rate density, it is necessary to calculate the $\mathrm{PBH}$ detectable volume for a given detector. In general, the expected number of photons detectable by an observatory on the Earth's surface from a PBH burst of duration $\tau$ at a non-cosmological distance $r$ and zenith angle $\theta$ is

$$
\mu(r, \theta, \tau)=\frac{(1-f)}{4 \pi r^{2}} \int_{E_{1}}^{E_{2}} \frac{d N(\tau)}{d E} A(E, \theta) d E
$$

where $f$ is the dead time fraction of the detector and $d N / d E$ is the black hole gamma ray spectrum integrated from remaining time $\tau$ to 0 . The values $E_{1}$ and $E_{2}$ correspond to the lower and upper bounds respectively of the energy range searched and $A(E, \theta)$ is the effective area of the detector as a function of photon energy and zenith angle. Typically the function $A(E, \theta)$ is obtained from a simulation of the detector. For Milagro and HAWC, 


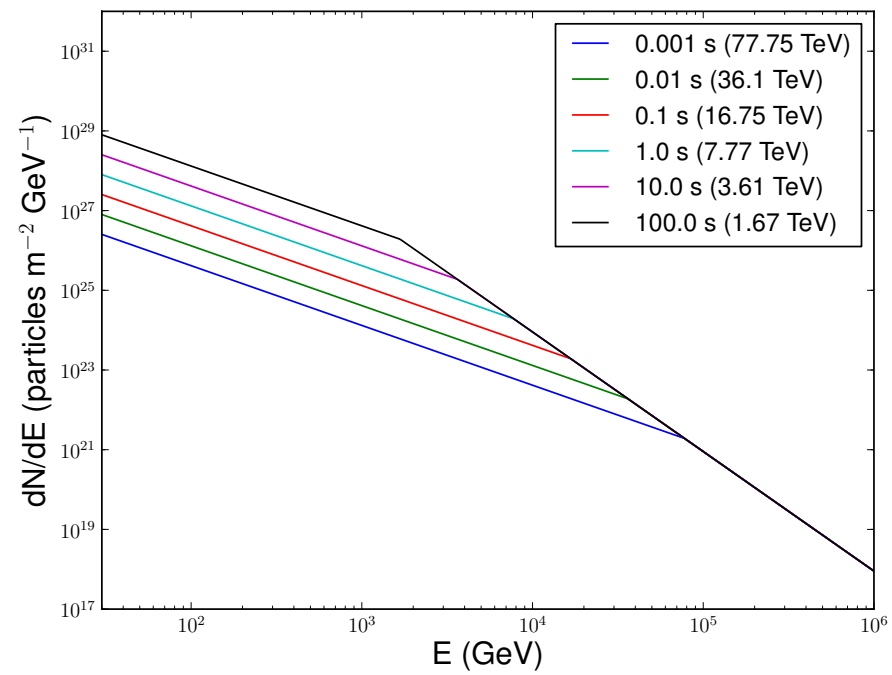

Figure 1: Time-integrated gamma-ray spectrum over various $\mathrm{PBH}$ remaining lifetimes using the parametrization of Equation 2. The black hole temperature at the start of observation is shown in parentheses.

the dependence of $A(E, \theta)$ on the zenith angle is usually given in discrete bands (represented by $\theta_{i}$ ). We will define these bands specifically for a given observatory in Section 4.

The background cosmic-ray flux at the Earth's surface is much higher than the background gamma-ray flux. Most events detected by EAS arrays such as Milagro or HAWC are air showers induced by cosmic rays. To search for the emission from a $\mathrm{PBH}$ burst one needs to look for an excess that cannot be explained by statistical fluctuations of the background.

In this paper, we estimate $\mu_{\circ}\left(\theta_{i}, \tau\right)$, the minimum number of counts needed for a detection for different burst durations $\tau$, by finding the number of counts required over the background for a $5 \sigma$ significance (after correcting for multiple trials). First, we calculate the background rates $\left(R\left(\theta_{i}\right)\right)$ utilizing a Monte Carlo simulation (for HAWC) or actual data (for Milagro). The background rate depends on the spatial bin-size, burst duration and background rejection parameters, as well as the zenith angle band $\theta_{i}$. In section 4 , we optimize these parameters to minimize the background rates and maximize the sensitivity. Using the background rates, we then find the $\mu_{\circ}\left(\theta_{i}, \tau\right)$ values required for a $50 \%$ probability of detecting a $5 \sigma$ excess after a given number of trials, based on the Poisson fluctuations of the signal 
around $\mu_{\circ}\left(\theta_{i}, \tau\right)$.

We define a $5 \sigma$ detection after correction for $N_{t}$ trials to be the number of counts $n$ which would have a Poisson probability $P$ corresponding to a Bonferroni corrected p-value $p_{c}$ given by

$$
p_{c}=p_{o} / N_{t}=P\left(\geq n \mid n_{\mathrm{bk}}\right) .
$$

Here, $p_{0}=2.3 \times 10^{-7}$ is the p-value corresponding to $5 \sigma, n_{\mathrm{bk}}=\tau R\left(\theta_{i}\right)$ is the number of background counts expected over the burst duration $\tau$, and $P\left(\geq n \mid n_{\mathrm{bk}}\right)$ denotes the Poisson probability of getting $n$ or more counts when the Poisson mean is $n_{\mathrm{bk}}$. We take the value of $\mu_{\circ}\left(\theta_{i}, \tau\right)$ to be the amount of expected signal which would satisfy this criterion $50 \%$ of the time. We find $\mu_{\circ}\left(\theta_{i}, \tau\right)$ by estimating the Poisson probability $P$ of finding at least $n$ counts to be $50 \%$ according to the relation

$$
P\left(\geq n \mid\left(n_{\mathrm{bk}}+\mu_{\circ}\left(\theta_{i}, \tau\right)\right)\right)=0.5 \text {. }
$$

By equating the values of $\mu_{\circ}\left(\theta_{i}, \tau\right)$ found from Equation 5 to $\mu\left(r, \theta_{i}, \tau\right)$ in Equation 3 and solving for $r$, we calculate the maximum distance from which a PBH burst could be detected by the high-energy observatory,

$$
r_{\max }\left(\theta_{i}, \tau\right)=\sqrt{\frac{(1-f)}{4 \pi \mu_{\circ}\left(\theta_{i}, \tau\right)} \int_{E_{1}}^{E_{2}} \frac{d N(\tau)}{d E} A\left(E, \theta_{i}\right) d E}
$$

for various zenith angle bands $\theta_{i}$ and burst durations $\tau$. Denoting the effective field-of-view of the detector for a given zenith band by

$$
\operatorname{FOV}_{\text {eff }}\left(\theta_{i}\right)=2 \pi\left(\cos \theta_{i, \min }-\cos \theta_{i, \max }\right) \mathrm{sr}
$$

where $\theta_{i, \min }$ and $\theta_{i, \max }$ are the minimum and maximum zenith angles in band $i$, the detectable volume is then

$$
V(\tau)=\sum_{i} V\left(\theta_{i}, \tau\right)=\frac{4}{3} \pi \sum_{i} r_{\max }^{3}\left(\theta_{i}, \tau\right) \frac{\mathrm{FOV}_{\mathrm{eff}}\left(\theta_{i}\right)}{4 \pi}
$$

\subsection{Upper Limit Estimation}

If the PBHs are uniformly distributed in the solar neighborhood, the X\% confidence level upper limit $\left(U L_{X}\right)$ on the rate density of PBHs bursts (that is, the number of bursts occurring locally per unit volume per unit time) can be estimated as

$$
U L_{X}=\frac{m}{V S}
$$


if at the $\mathrm{X} \%$ confidence level the detector observes zero bursts. Here $V$ is the effective detectable volume from which a PBH can be detected, $S$ is the total search duration and $m$ is the upper limit on the expected number of $\mathrm{PBH}$ bursts given that at the $\mathrm{X} \%$ confidence level zero bursts are observed at Earth. Note that for Poisson fluctuations $P(0 \mid m)=1-X$ and so $m=$ $\ln (1 /(1-X))$. If $X=99 \%$ and $m=\ln 100 \approx 4.6$, the upper limit on the $\mathrm{PBH}$ burst rate density will be

$$
U L_{99}=\frac{4.6}{V S}
$$

\section{Results}

\subsection{Milagro Limits on the Rate-Density of PBH Bursts}

During the early days of its operation, Milagro had lower angular resolution prior to the addition of the outrigger array and used a triggering system that did not accept many of the low energy events. Thus, for this search we used the last five years of Milagro data: specifically from 03/01/2003 to 03/01/2008. (Due to various detector-related issues, $7 \%$ of the data taken during this period was also not used.) Selection cuts were made to increase the quality of the data searched. Reconstructed events which have a predicted angular reconstruction error greater than $2^{\circ}$ were rejected. (This corresponds to $n$ fit $>20$ where $n$ fit is the number of PMTs participating in the reconstruction of the shower.) The maximum zenith angle used was $45^{\circ}$ and the best limits were obtained with no gamma-hadron separation cut applied, because such a cut also strongly lowered the Milagro photon effective area at energies below $1 \mathrm{TeV}$. Overall, our analysis utilized 1673 days (4.58 years) worth of good data, amounting to $\sim 93 \%$ of the total Milagro data collected during the five year period (neglecting the dead time). The Milagro search and its optimization presented here are described in further detail in [17].

We performed a blind search (that is, a search utilizing no external triggers) for burst durations ranging from $250 \mu$ s to 6 minutes. First we created skymaps for overlapping time intervals, each offset by $10 \%$ of the preset burst duration. We then spatially binned the skymap and searched for locations with significant excess over background in the Milagro data. The optimum bin-size was determined using a Monte-Carlo simulation and varied with the pre-set burst duration. For short durations the optimum bin size was of order $\sim 1.8^{\circ}$ and for long durations it was $\sim 0.8^{\circ}$ : for short durations, Milagro was signal-limited requiring a larger bin-size to accumulate more 


\section{curves for the selected three zenith angle bands.}

\begin{tabular}{|c|c|c|c|}
\hline Zenith Angle Band $\theta_{i}$ & $a_{i}$ & $b_{i}$ & $c_{i}$ \\
\hline $0^{\circ}-15^{\circ}\left(\theta_{1}\right)$ & -0.4933 & 4.7736 & -2.4272 \\
\hline $15^{\circ}-30^{\circ}\left(\theta_{2}\right)$ & -0.5037 & 5.0102 & -3.4015 \\
\hline $30^{\circ}-45^{\circ}\left(\theta_{3}\right)$ & -0.4273 & 4.7931 & -4.3030 \\
\hline
\end{tabular}

Table 1: Milagro effective area parametrization for various zenith angle bands. a more restricted bin-size to reduce background contamination.

No statistically significant $(5 \sigma)$ event was observed over the 4.58 years of data. Proceeding on the basis of null detection, we calculated the upper limits on the $\mathrm{PBH}$ burst rate density following the methodology described in Section 3. For Milagro, we parameterized the effective area as $A\left(E, \theta_{i}\right)=$ $10^{a_{i}(\log E)^{2}+b_{i} \log E+c_{i}} \mathrm{~m}^{2}$ with the parameters $a_{i}, b_{i}$ and $c_{i}$ given in Table 1 for three zenith angle bands. Figure 2 shows the Milagro effective area

signal; for long durations, Milagro became background-dominated, requiring

Table 1: Milagro effective area parametrization for various zenith angle bands.

We utilized a Monte Carlo simulation to calculate the $\mu_{\circ}\left(\theta_{i}, \tau\right)$ values for various burst durations. Because the trials in our search were not independent, we took $N_{t}$ in Equation 4 to be the effective number of independent trials calculated using the method described in [17]. The effective number of independent trials ranged from $\sim 0.1 \%$ to $\sim 40 \%$ of the total number of trials depending on the search duration, with the shorter search durations having the lower fraction of effective trials. The dependence of the limit on the estimated number of independent trials is quite mild $\left(\sim N_{t}^{0.018}\right)$ so that varying the estimated trials by 3 orders of magnitude produces less than $15 \%$ change in the limit. The resulting $\mu_{\circ}\left(\theta_{i}, \tau\right)$ values are listed in Table 2. These $\mu_{\circ}\left(\theta_{i}, \tau\right)$ values and the Milagro effective area parameterizations were then used to derive the maximum detectable distance of a $\mathrm{PBH}$ burst, $r_{\max }\left(\theta_{i}, \tau\right)$, using Equation 6 assuming an energy range of $E_{1}=50$ $\mathrm{GeV}$ and $E_{2}=100 \mathrm{TeV}$ and a deadtime of $7 \%$. The derived $r_{\max }\left(\theta_{i}, \tau\right)$ values were then used to calculate the effective volume that was probed by the Milagro observatory. Using Equation 10, we calculated 99\% upper limits for various $\mathrm{PBH}$ remaining lifetimes and the effective total search period of 4.58 years. Our results are shown in Table 2 and in Figure 3. According to our results, Milagro is most sensitive to burst durations of about $1 \mathrm{~s}$. For shorter durations, the Milagro data is starved for signal photons; for longer durations, the background tends to dominate the signal. We note that Milagro has a systematic flux uncertainty of $\sim 30 \%$ [18] which translates into an $\sim 50 \%$ uncertainty in our calculated limit (shown as a pink shaded band 
in Figure 3).

\begin{tabular}{|l|c|c|}
\hline Burst Duration $\tau(\mathrm{s})$ & $\mu_{\circ, \tau}$ & $U L_{99}\left(\mathrm{pc}^{-3} \mathrm{yr}^{-1}\right)$ \\
\hline 0.001 & 11 & $3.1 \times 10^{5}$ \\
\hline 0.01 & 16 & $1.2 \times 10^{5}$ \\
\hline 0.1 & 22 & $5.4 \times 10^{4}$ \\
\hline 1.0 & 35 & $3.6 \times 10^{4}$ \\
\hline 10.0 & 65 & $3.8 \times 10^{4}$ \\
\hline 100.0 & 150 & $6.9 \times 10^{4}$ \\
\hline
\end{tabular}

Table 2: Counts $\left(\mu_{\circ}(\tau)\right)$ needed over the background for a $5 \sigma$ detection with $99 \%$ probability and calculated $99 \%$ confidence upper limits $\left(U L_{99}\right)$ for various burst durations $(\tau)$ for Milagro.

\subsection{Improved HAWC Sensitivity to the Rate Density of PBH Bursts}

Milagro's successor HAWC is located at a higher altitude and features a better detector design, allowing for superior sensitivity to PBH bursts. In this section, we apply our methodology to estimate the HAWC sensitivity to PBH bursts. In our calculations, all relevant characteristics of the HAWC detector are encoded in the effective area. We calculate the effective area using a Monte Carlo simulation which models the interaction of photons and cosmic rays in the atmosphere and the response of the detector to the extensive air showers generated by these particles. The effective area is then defined as the ratio of the number of events that satisfies a given set of cuts to the total number of events multiplied by the total throw area of the Monte Carlo simulation. In our case the Monte Carlo throw area is a circle of $1000 \mathrm{~m}$ radius. The cuts are comprised of a trigger cut, an angle cut and a gamma-hadron separation cut. For the trigger, HAWC will use events with $n$ Hit, the number of PMTs hit by the air shower, greater than a certain value. The angle cut is employed to specify the direction of the photons and is a measure of HAWC's angular resolution. In the simulated events we use an angular parameter DelAngle which is the difference between the true location of the particle in the sky and the reconstructed sky location of the particle. This is a proxy for the angular search bin-size. Because the background is predominantly protons, the angle cut was not used to calculate the HAWC effective area for protons and the gamma-hadron separation cut was used to reduce background events. The standard gamma-hadron separation parameter for HAWC is called compactness and is defined as $n H i t / C x P E 40$ where $C x P E 40$ is the number of photoelectrons recorded in 


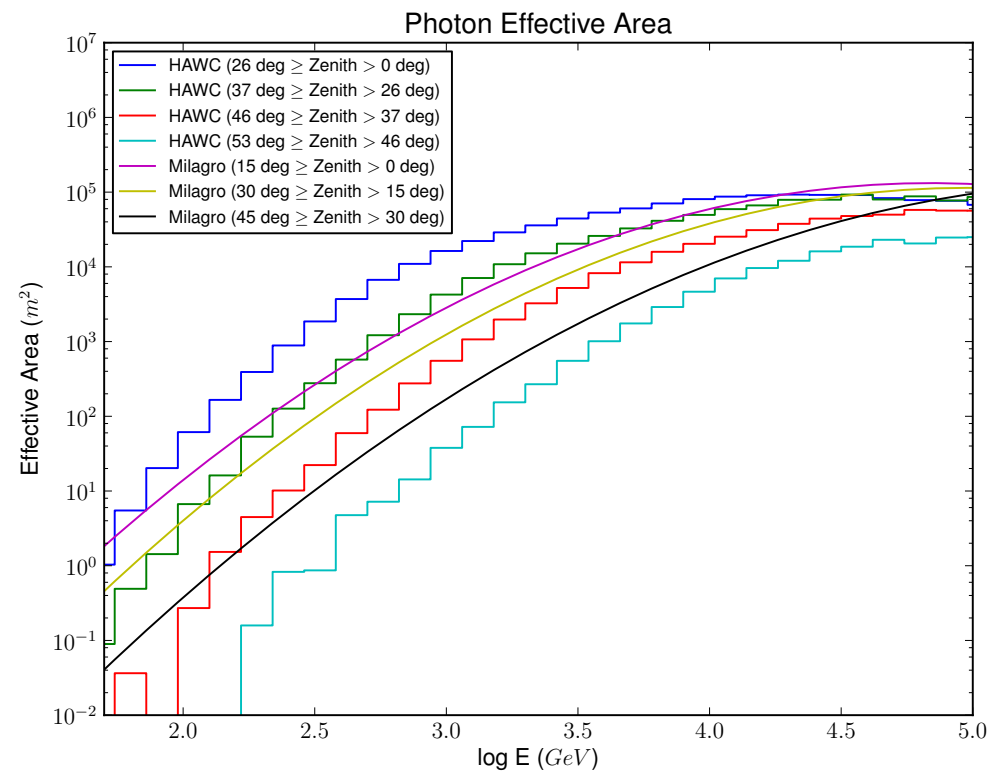

Figure 2: Effective Area for photon detection for HAWC and Milagro as a function of energy. The Milagro effective area curves use $n$ Hit $>50$, Del Angle $<1.5$ deg and no gammahadron separation cuts. The HAWC effective area curves use $n$ Hit $>100$, DelAngle $<0.8$ $\operatorname{deg}$ and $n H i t / C x P E 40>7$. The HAWC cuts are optimized for the PBH spectrum and utilize an $n$ Hit cut that is well above the intrinsic threshold. This and the fact that Milagro used no gamma-hadron cut result in an effective area for Milagro which is larger than for HAWC at low energies. However, HAWC has superior sensitivity in the PBH search.

the strongest hit PMT outside a $40 \mathrm{~m}$ radius from the reconstructed shower core. The shower core represents the location on the ground where the original particle would have hit had it not interacted with the atmosphere. Figure 2 shows the HAWC effective area $A\left(E, \theta_{i}\right)$ for photons as a function of photon energy and zenith angle band using the optimum cuts for a $10 \mathrm{~s}$ burst search $(n H i t>100$, DelAngle $<0.8 \mathrm{deg}$, and with $n$ Hit $/ C x P E 40>$ 7).

In order to estimate the background rate $R_{b}(\theta, \xi)$ where $\xi$ is a measure of the spatial resolution of HAWC, we used the ATIC cosmic ray spectrum given in Reference [19]

$$
\frac{d N_{p}}{d E}=7900 \times\left(\frac{E}{1 \mathrm{GeV}}\right)^{-2.65} \quad \text { particles } \mathrm{m}^{-2} \mathrm{~s}^{-1} \mathrm{sr}^{-1} \mathrm{GeV}^{-1}
$$

and convolved it with the HAWC proton effective area for a given zenith 
angle band,

$$
R_{b}\left(\theta_{i}, \xi\right)=\int_{E_{1}}^{E_{2}} \frac{d N_{p}}{d E} A_{p}\left(E, \theta_{i}\right) d E \times 2 \pi(1.0-\cos (\xi)) \times 1.2
$$

In Equation 12, the $2 \pi(1.0-\cos (\xi))$ term represents the patch in the sky that corresponds to the spatial resolution of HAWC in steradians. In our case $\xi=$ DelAngle. The factor 1.2 is a correction factor incorporating the other particle species in the cosmic ray background.

\begin{tabular}{|l|l|}
\hline Parameter & Values \\
\hline$n$ Hit $\geq$ & $30,70,100,150,200,250$ \\
\hline DelAngle $\leq$ & $0.1^{\circ}, 0.3^{\circ}, 0.8^{\circ}, 1.0^{\circ}, 3.0^{\circ}, 8.0^{\circ}$ \\
\hline Compactness $\geq$ & $1,2,3,4,5,6,7,8,9,10$ \\
\hline
\end{tabular}

Table 3: Various parameter cuts used for the simple parameter search.

Because we seek the sensitivity in the case where there is no prior knowledge of the burst location, we need to take into account the number of trials performed for the search. For example, if the HAWC field-of-view (1.8 sr) is divided into spatial bins of radius $0.7^{\circ}$, then there will be approximately $10^{4}$ spatial bins (trials) per time bin searched. The optimum spatial binsize depends on the search duration, the trigger criteria, and the value of the gamma-hadron separation parameter. The number of time bins is estimated by dividing the total search period (estimated as 5 years for HAWC) by the burst duration $\tau$. Thus the total number of trials depends on the burst duration $\tau$, the optimal spatial bin-size DelAngle, the trigger criterion $n$ Hit and the value of the compactness parameter. In order to find the optimum set of cuts, we performed a simple parameter search and identified the set of values which give the best PBH limit according to the method described in Section 3.

For burst durations ranging from $0.001 \mathrm{~s}$ to $100 \mathrm{~s}$, we performed cuts on all parameter combinations given in Table 3 on the Monte Carlo output and calculated corresponding effective areas for photons and protons. Using Equations 11 and 12, we then calculated the background rate and the background number density $n_{\mathrm{bk}}\left(\theta_{i}, \xi\right)=\tau R_{b}\left(\theta_{i}, \xi\right)$ (see Equation 4 ) which depends on the zenith angle band and the spatial resolution. As remarked earlier, the effective number of independent trials differs for each parameter combination. Taking this into account, we have calculated the $\mu_{\circ}\left(\theta_{i}, \tau\right)$ values corresponding to burst durations ranging from $0.001 \mathrm{~s}$ to $100 \mathrm{~s}$ for 


\begin{tabular}{|l|c|c|c|}
\hline Duration $\tau(\mathrm{s})$ & nHit & DelAngle $(\mathrm{deg})$ & Compactness \\
\hline 0.001 & 30 & 3.0 & 3 \\
\hline 0.01 & 30 & 3.0 & 3 \\
\hline 0.1 & 70 & 1.0 & 4 \\
\hline 1.0 & 70 & 1.0 & 5 \\
\hline 10.0 & 100 & 0.8 & 7 \\
\hline 100.0 & 100 & 0.8 & 7 \\
\hline
\end{tabular}

Table 4: Optimized cuts for various burst durations.

various zenith angle bands. These $\mu_{\circ}\left(\theta_{i}, \tau\right)$ values and the effective area for photons are then inserted into Equation 6 (with $E_{1}=50 \mathrm{GeV}$ and $E_{2}=100$ $\mathrm{TeV})$ and the maximum distances $r_{\max }\left(\theta_{i}, \tau\right)$ at which a PBH burst can be detected by the HAWC observatory is calculated for various burst durations $\tau$ assuming negligible dead time. Using these $r_{\max }\left(\theta_{i}, \tau\right)$ values and Equation 8 , we have determined the effective detectable volume $V(\tau)$ for each burst duration. The PBH limit for each parameter combination was calculated using Equation 10 and the set of cuts that gives the best limit for a given burst duration was selected. The resulting optimized parameter cut values are given in Table 4 . The corresponding values of $\mu_{\circ}\left(\theta_{i}, \tau\right)$ for a $5 \sigma$ detection are given in Table 5 with the associated background counts and number of trials factor.

The final 99\% confidence level upper limits on the $\mathrm{PBH}$ burst rate density are given in Table 6, assuming zero PBH bursts are observed over the 5 year HAWC search period. For each burst duration, the maximum detectable distance for zenith angle band $\theta_{1}$ and the corresponding effective detectable volume are also shown in Table 6 . We note that HAWC systematic uncertainties have not been included in this study. In Figure 3, the blue, green, and red thin dashed curves denote the $\mathrm{PBH}$ rate density upper limits that HAWC will set if zero PBH bursts are detected over a one, two and five year search period respectively. Upper limits based on earlier null detections from various other observatories are also shown in Figure $3[20 ; 21 ; 22 ; 23 ; 10]$. All limits shown in Figure 3 were obtained based on the PBH Standard Emission Model $[11 ; 6]$. 


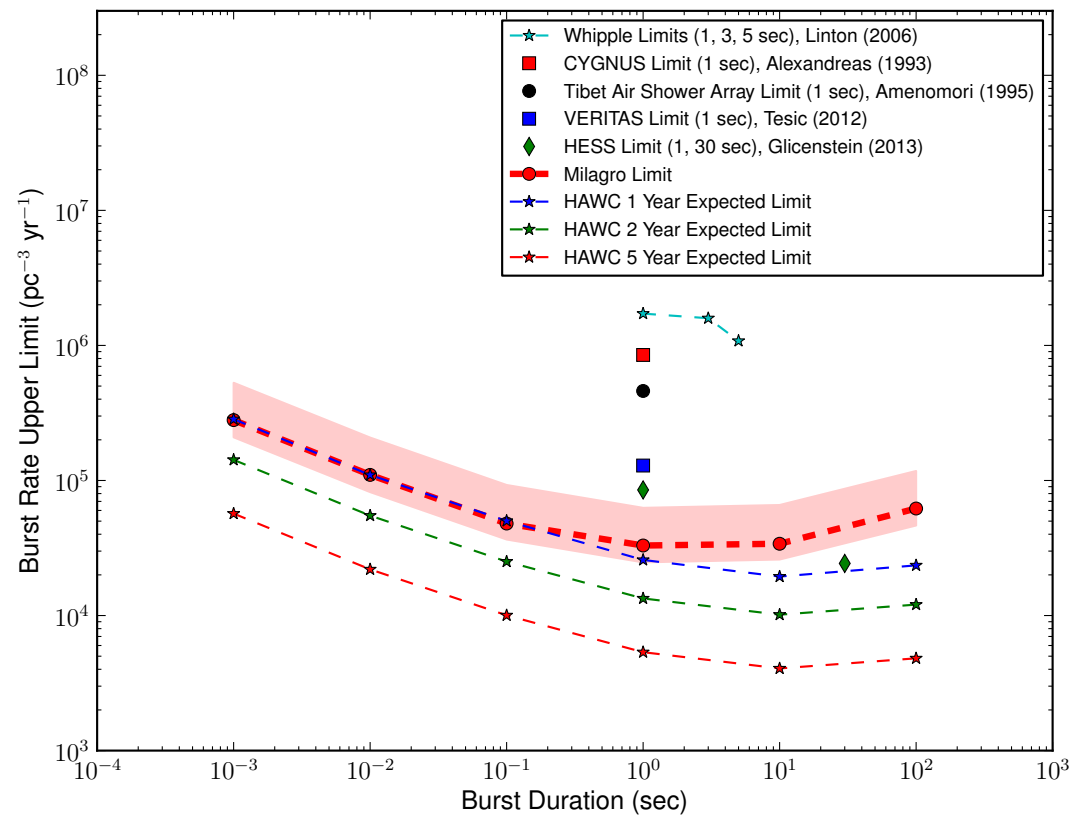

Figure 3: PBH Burst Rate Density Upper Limits from Milagro and projected for HAWC, compared with limits from previous direct search experiments $[20 ; 21 ; 22 ; 23 ; 10]$. The pink band represents the $50 \%$ systematic uncertainty of the Milagro limit. All limits are at the 99\% Confidence Level (we have rescaled the reported 95\% CL HESS limit to 99\% CL) and were obtained based on the PBH Standard Emission Model. 


\begin{tabular}{|l|c|c|c|c|}
\hline Duration $\tau(\mathrm{s})$ & Zenith Angle Band $\theta_{i}$ & Number of Trials & Bgnd. Counts $n_{\mathrm{bk}}$ & $\mu_{\circ}\left(\theta_{i}, \tau\right)$ \\
\hline 0.001 & $0^{\circ}-26^{\circ}\left(\theta_{1}\right)$ & $3.3 \times 10^{13}$ & 0.0637 & 10.6 \\
\hline 0.001 & $26^{\circ}-37^{\circ}\left(\theta_{2}\right)$ & $3.3 \times 10^{13}$ & 0.0240 & 8.6 \\
\hline 0.001 & $37^{\circ}-46^{\circ}\left(\theta_{3}\right)$ & $3.3 \times 10^{13}$ & 0.0083 & 7.7 \\
\hline 0.001 & $46^{\circ}-53^{\circ}\left(\theta_{4}\right)$ & $3.3 \times 10^{13}$ & 0.0026 & 6.7 \\
\hline 0.01 & $0^{\circ}-26^{\circ}\left(\theta_{1}\right)$ & $3.3 \times 10^{12}$ & 0.6372 & 17.0 \\
\hline 0.01 & $26^{\circ}-37^{\circ}\left(\theta_{2}\right)$ & $3.3 \times 10^{12}$ & 0.2397 & 13.4 \\
\hline 0.01 & $37^{\circ}-46^{\circ}\left(\theta_{3}\right)$ & $3.3 \times 10^{12}$ & 0.0832 & 10.6 \\
\hline 0.01 & $46^{\circ}-53^{\circ}\left(\theta_{4}\right)$ & $3.3 \times 10^{12}$ & 0.0256 & 8.6 \\
\hline 0.1 & $0^{\circ}-26^{\circ}\left(\theta_{1}\right)$ & $3.0 \times 10^{12}$ & 0.1355 & 11.5 \\
\hline 0.1 & $26^{\circ}-37^{\circ}\left(\theta_{2}\right)$ & $3.0 \times 10^{12}$ & 0.0456 & 9.6 \\
\hline 0.1 & $37^{\circ}-46^{\circ}\left(\theta_{3}\right)$ & $3.0 \times 10^{12}$ & 0.0144 & 7.7 \\
\hline 0.1 & $46^{\circ}-53^{\circ}\left(\theta_{4}\right)$ & $3.0 \times 10^{12}$ & 0.0036 & 6.7 \\
\hline 1.0 & $0^{\circ}-26^{\circ}\left(\theta_{1}\right)$ & $3.0 \times 10^{11}$ & 1.0481 & 18.6 \\
\hline 1.0 & $26^{\circ}-37^{\circ}\left(\theta_{2}\right)$ & $3.0 \times 10^{11}$ & 0.3422 & 14.3 \\
\hline 1.0 & $37^{\circ}-46^{\circ}\left(\theta_{3}\right)$ & $3.0 \times 10^{11}$ & 0.1055 & 10.6 \\
\hline 1.0 & $46^{\circ}-53^{\circ}\left(\theta_{4}\right)$ & $3.0 \times 10^{11}$ & 0.0251 & 8.6 \\
\hline 10.0 & $0^{\circ}-26^{\circ}\left(\theta_{1}\right)$ & $4.6 \times 10^{10}$ & 2.4405 & 23.2 \\
\hline 10.0 & $26^{\circ}-37^{\circ}\left(\theta_{2}\right)$ & $4.6 \times 10^{10}$ & 0.7039 & 16.0 \\
\hline 10.0 & $37^{\circ}-46^{\circ}\left(\theta_{3}\right)$ & $4.6 \times 10^{10}$ & 0.1912 & 11.5 \\
\hline 10.0 & $46^{\circ}-53^{\circ}\left(\theta_{4}\right)$ & $4.6 \times 10^{10}$ & 0.0451 & 8.6 \\
\hline 100.0 & $0^{\circ}-26^{\circ}\left(\theta_{1}\right)$ & $4.6 \times 10^{9}$ & 24.4049 & 51.3 \\
\hline 100.0 & $26^{\circ}-37^{\circ}\left(\theta_{2}\right)$ & $4.6 \times 10^{9}$ & 7.0394 & 31.6 \\
\hline 100.0 & $37^{\circ}-46^{\circ}\left(\theta_{3}\right)$ & $4.6 \times 10^{9}$ & 1.9118 & 20.8 \\
\hline 100.0 & $46^{\circ}-53^{\circ}\left(\theta_{4}\right)$ & $4.6 \times 10^{9}$ & 0.4513 & 14.2 \\
\hline
\end{tabular}

Table 5: Counts $\mu_{\circ}\left(\theta_{i}, \tau\right)$ needed over the background for a $5 \sigma$ detection with $50 \%$ probability for various burst durations and zenith angle bands for HAWC.

\begin{tabular}{|l|c|c|c|}
\hline Burst Duration $\tau(\mathrm{s})$ & $r_{\max }(\mathrm{pc})$ & Effective Volume $V(\tau)\left(\mathrm{pc}^{3}\right)$ & PBH Upper Limit $\left(\mathrm{pc}^{-3} \mathrm{yr}^{-1}\right)$ \\
\hline 0.001 & 0.033 & 0.000016 & 56861 \\
\hline 0.01 & 0.044 & 0.000042 & 21976 \\
\hline 0.1 & 0.062 & 0.000092 & 10038 \\
\hline 1.0 & 0.078 & 0.000172 & 5354 \\
\hline 10.0 & 0.089 & 0.000227 & 4059 \\
\hline 100.0 & 0.085 & 0.000191 & 4822 \\
\hline
\end{tabular}

Table 6: The maximum detectable distance (for zenith band $\theta_{1}$ ), the detectable effective volume and HAWC PBH limit in the event of null detection in 5 years for various remaining PBH lifetimes. 


\section{Discussion}

In this work, we report new $\mathrm{PBH}$ burst rate density upper limits on parsec scales based on a direct search performed with Milagro data. These new Milagro limits probe various burst timescales previously not investigated. For $1 \mathrm{~s}$ bursts, which were probed by numerous earlier experiments, the Milagro limit is now the most constraining. Only the HESS limit at $30 \mathrm{~s}$ is more constraining than the Milagro limits. Milagro's successor, the HAWC observatory, will be even more sensitive to PBH bursts. As seen in Figure 3, a null detection from a 5 year search with the HAWC Observatory will set $\mathrm{PBH}$ upper limits which are significantly better than the upper limits set by any previous PBH burst search including Milagro. Also note that HAWC will surpass the current HESS best limit for a $30 \mathrm{~s}$ burst in one year. According to our study, if a PBH explodes within 0.074 parsec $(15,000 \mathrm{AU})$ of Earth and within 26 degrees of zenith, HAWC will have a $95 \%$ probability of detecting it at $5 \sigma$ (as optimized in a $10 \mathrm{~s}$ search after trials corrections). HAWC would see with $95 \%$ probability a $\mathrm{PBH}$ burst within 37 degrees of zenith if it happens within 0.058 parsec $(12,000 \mathrm{AU})$ of Earth.

Direct search limits are limits on the local distribution of very low mass black holes, regardless of their initial mass or their formation era and mechanism. A $10^{9} \mathrm{~g}$ black hole has a remaining evaporation lifetime of $1 \mathrm{~s}$ and a $7 \times 10^{11} \mathrm{~g}$ black hole has a remaining evaporation lifetime of 5 years. It can be shown that the evaporation rate [15] of an individual black hole whose remaining lifetime is much less than the age of the universe implies that the number density function of any population of BHs with present masses $\sim M<<5 \times 10^{14} \mathrm{~g}$ has the form $d n / d M \propto M^{2}$ around $M$ [24]. From Figure 3 , a null detection from a 5 year HAWC search would correspond to an upper limit on the number of local bursts of less than $2.0 \times 10^{4} \mathrm{pc}^{-3}$ over 5 years and hence to an upper limit on the local density in $7 \times 10^{11} \mathrm{~g}$ or lighter black holes of $\rho(\leq M) \lesssim 5 \times 10^{-18} M_{\odot} \mathrm{pc}^{-3}$. This applies to very small black holes produced in the present universe as well as primordial black holes. This limit is well below the total (visible and dark matter) local dynamical mass density in the solar neighborhood (the Oort limit) determined from Hipparcos satellite measurements, $\rho_{\odot, \text { Oort }}=0.102( \pm 0.010) M_{\odot} \mathrm{pc}^{-3}[25]$, and the local dark matter density in the solar neighborhood, $\rho_{\odot, D M}=0.008( \pm 0.003) M_{\odot}$ $\mathrm{pc}^{-3}[26]$. If the present number density function for such small black holes, $d n / d M \propto M^{2}$, can be extrapolated to black holes of present mass $5 \times 10^{14} \mathrm{~g}$, a null detection from a 5 year HAWC search would correspond to an upper limit on the local density in $5 \times 10^{14} \mathrm{~g}$ black holes of $\rho(\leq M) \lesssim 10^{-6} M_{\odot}$ $\mathrm{pc}^{-3}$, five orders of magnitude less than $\rho_{\odot, \text { Oort }}$. 
Direct search limits on the local rate density of $\mathrm{PBH}$ bursts are higher than the average cosmological $\mathrm{PBH}$ burst rate density limit implied by the $100 \mathrm{MeV}$ extragalactic gamma-ray background constraint on the emission of $5 \times 10^{14} \mathrm{~g}$ black holes $[7 ; 27]^{1}$. However, PBHs have the gravitational properties of cold dark matter and so should be clustered in our Galaxy, enhancing the local PBH burst rate density by many orders of magnitude over the average cosmological rate [28]. Thus a substantial number of PBHs that evaporate producing gamma ray bursts may exist in our Galaxy. PBHs clustered in our Galactic halo should also contribute an anisotropic Galactic gamma-ray background, separable from the extragalactic gamma-ray background. Wright claims that such a halo background has been detected [8]. The direct search limits are also weaker than the average Galactic PBH burst rate density limit on kiloparsec scales implied by the Galactic antiproton background [9] but the antiproton-derived limit depends on the assumed $\mathrm{PBH}$ distribution, the propagation of antiprotons within the Galaxy, and the secondary antiproton component. Direct search limits are independent of assumptions concerning the PBH spatial distribution.

In deriving the new limits, we have assumed the Standard Evaporation Model in which the Hawking-radiated particles leave the vicinity of the black hole without substantially interacting with other Hawking-radiated particles. As shown in detail in [3], the energy of the Hawking-radiated particles and the time interval between subsequent emissions are such that selfinteractions between the Hawking-radiated particles or their decay products should not form a QED or QCD photosphere around the evaporating black hole nor modify the predicted $\mathrm{PBH}$ gamma ray burst spectra. However, if the $\mathrm{PBH}$ is embedded in an ambient high density plasma or strong magnetic field, interactions may arise with the net effect that the predicted $\mathrm{PBH}$ burst gamma ray spectrum may be enhanced at low energies and decreased at high energies. Because of the remaining BH lifetime's $\tau \propto T^{-3}$ dependence (see Equation 1), as-yet unknown particle modes manifesting at high temperatures are unlikely to substantially modify the predicted spectra by more than a factor of 2 . An exception would be the low temperature Hagedorn Model in which all of the final burst emission is produced at a black hole temperature close to the QCD confinement scale, $\Lambda_{Q C D} \simeq 250-300$ $\mathrm{MeV}$ [7]. The low temperature Hagedorn Model would generate a stronger

\footnotetext{
${ }^{1}$ We note that all limits on the expected burst rate derived from the extragalactic or galactic gamma ray or antiproton backgrounds have assumed an extrapolation of the form $d n / d M \propto M^{2}$ from masses of $5 \times 10^{14} \mathrm{~g}$ down to the very small masses of presently bursting PBHs.
} 
burst in gamma rays detectable at lower energies but is not consistent with the QCD behaviour observed in terrestrial accelerators [3].

In conclusion, the HAWC observatory has the ability to directly detect emission from nearby PBH bursts. This capability is scientifically very important, given the large number of early universe theories that predict $\mathrm{PBH}$ formation and the uncertainty in the degree to which PBHs may cluster locally. A confirmed direct detection of an evaporating $\mathrm{PBH}$ would also provide unparalleled insight into general relativity and high energy particle physics.

\section{Acknowledgments}

We gratefully acknowledge Scott DeLay his dedicated efforts in the construction and maintenance of the HAWC experiment. This work has been supported by: the National Science Foundation, the US Department of Energy Office of High-Energy Physics, the LDRD program of Los Alamos National Laboratory, Consejo Nacional de Ciencia y Tecnologia (grants 55155, 103520, 105033, 105666, 122331 and 132197), Red de Fisica de Altas Energias, DGAPA-UNAM (grants IN105211, IN108713 and IN121309, IN115409), VIEP-BUAP (grant 161- EXC-2011), the University of Wisconsin Alumni Research Foundation, the Luc Binette Foundation UNAM Postdoctoral Fellowship program and the Institute of Geophysics and Planetary Physics at Los Alamos National Lab. Many of us are grateful for inspiring discussions with the late Donald Coyne on the subject of primordial black holes. Finally, we thank the anonymous referee for comments that significantly improved the paper.

\section{References}

[1] B. J. Carr, K. Kohri, Y. Sendouda, and J. Yokoyama, "New cosmological constraints on primordial black holes," Phys. Rev. D, vol. 81, p. 104019, May 2010.

[2] S. W. Hawking, "Black hole explosions?," Nature, vol. 248, pp. 30-31, Mar. 1974.

[3] J. H. MacGibbon, B. J. Carr, and D. N. Page, "Do evaporating black holes form photospheres?," Phys. Rev. D, vol. 78, p. 064043, Sept. 2008.

[4] B. J. Carr, "Primordial Black Holes: Do They Exist and Are They Useful?," ArXiv Astrophysics e-prints, Nov. 2005. 
[5] N. Gehrels and P. Mészáros, "Gamma-Ray Bursts," Science, vol. 337, pp. 932-, Aug. 2012.

[6] F. Halzen, E. Zas, J. H. MacGibbon, and T. C. Weekes, "Gamma rays and energetic particles from primordial black holes," Nature, vol. 353, pp. 807-815, Oct. 1991.

[7] D. N. Page and S. W. Hawking, "Gamma rays from primordial black holes," ApJ., vol. 206, pp. 1-7, May 1976.

[8] E. L. Wright, "On the Density of Primordial Black Holes in the Galactic Halo," ApJ., vol. 459, p. 487, Mar. 1996.

[9] K. Abe, H. Fuke, S. Haino, T. Hams, M. Hasegawa, A. Horikoshi, K. C. Kim, A. Kusumoto, M. H. Lee, Y. Makida, S. Matsuda, Y. Matsukawa, J. W. Mitchell, J. Nishimura, M. Nozaki, R. Orito, J. F. Ormes, K. Sakai, M. Sasaki, E. S. Seo, R. Shinoda, R. E. Streitmatter, J. Suzuki, K. Tanaka, N. Thakur, T. Yamagami, A. Yamamoto, T. Yoshida, and K. Yoshimura, "Measurement of the Cosmic-Ray Antiproton Spectrum at Solar Minimum with a Long-Duration Balloon Flight over Antarctica," Physical Review Letters, vol. 108, p. 051102, Feb. 2012.

[10] J. Glicenstein, A. Barnacka, M. Vivier, T. Herr, and for the H. E. S. S. Collaboration, "Limits on Primordial Black Hole evaporation with the H.E.S.S. array of Cherenkov telescopes," ArXiv e-prints, July 2013.

[11] J. H. MacGibbon and B. R. Webber, "Quark- and gluon-jet emission from primordial black holes: The instantaneous spectra," Phys. Rev. D, vol. 41, pp. 3052-3079, May 1990.

[12] R. Atkins, W. Benbow, D. Berley, M.-L. Chen, D. G. Coyne, R. S. Delay, B. L. Dingus, D. E. Dorfan, R. W. Ellsworth, C. Espinoza, D. Evans, A. Falcone, L. Fleysher, R. Fleysher, G. Gisler, J. A. Goodman, T. J. Haines, C. M. Hoffman, S. Hugenberger, L. A. Kelley, S. Klein, I. Leonor, J. Macri, M. McConnell, J. F. McCullough, J. E. McEnery, R. S. Miller, A. I. Mincer, M. F. Morales, M. M. Murray, P. Nemethy, G. Paliaga, J. M. Ryan, M. Schneider, B. Shen, A. Shoup, G. Sinnis, A. J. Smith, G. W. Sullivan, T. N. Thompson, O. T. Tumer, K. Wang, M. O. Wascko, S. Westerhoff, D. A. Williams, T. Yang, and G. B. Yodh, "Milagrito, a TeV air-shower array," Nuclear Instruments and Methods in Physics Research A, vol. 449, pp. 478-499, July 2000. 
[13] HAWC Collaboration, A. U. Abeysekara, R. Alfaro, C. Alvarez, J. D. Álvarez, R. Arceo, J. C. Arteaga-Velázquez, H. A. Ayala Solares, A. S. Barber, B. M. Baughman, N. Bautista-Elivar, E. Belmont, S. Y. BenZvi, D. Berley, M. Bonilla Rosales, J. Braun, R. A. Caballero-Lopez, K. S. Caballero-Mora, A. Carramiñana, M. Castillo, U. Cotti, J. Cotzomi, E. de la Fuente, C. De León, T. DeYoung, R. Diaz Hernandez, J. C. Díaz-Vélez, B. L. Dingus, M. A. DuVernois, R. W. Ellsworth, A. Fernandez, D. W. Fiorino, N. Fraija, A. Galindo, F. Garfias, L. X. González, M. M. González, J. A. Goodman, V. Grabski, M. Gussert, Z. Hampel-Arias, C. M. Hui, P. Hüntemeyer, A. Imran, A. Iriarte, P. Karn, D. Kieda, G. J. Kunde, A. Lara, R. J. Lauer, W. H. Lee, D. Lennarz, H. León Vargas, E. C. Linares, J. T. Linnemann, M. Longo, R. Luna-GarcIa, A. Marinelli, H. Martinez, O. Martinez, J. Martínez-Castro, J. A. J. Matthews, P. Miranda-Romagnoli, E. Moreno, M. Mostafá, J. Nava, L. Nellen, M. Newbold, R. NoriegaPapaqui, T. Oceguera-Becerra, B. Patricelli, R. Pelayo, E. G. PérezPérez, J. Pretz, C. Rivière, D. Rosa-González, H. Salazar, F. Salesa, F. E. Sanchez, A. Sandoval, E. Santos, M. Schneider, S. Silich, G. Sinnis, A. J. Smith, K. Sparks, R. W. Springer, I. Taboada, P. A. Toale, K. Tollefson, I. Torres, T. N. Ukwatta, L. Villaseñor, T. Weisgarber, S. Westerhoff, I. G. Wisher, J. Wood, G. B. Yodh, P. W. Younk, D. Zaborov, A. Zepeda, and H. Zhou, "The HAWC Gamma-Ray Observatory: Sensitivity to Steady and Transient Sources of Gamma Rays," ArXiv e-prints, Sept. 2013.

[14] A. U. Abeysekara, J. A. Aguilar, S. Aguilar, R. Alfaro, E. Almaraz, C. Álvarez, J. d. D. Álvarez-Romero, M. Álvarez, R. Arceo, J. C. Arteaga-Velázquez, C. Badillo, A. Barber, B. M. Baughman, N. Bautista-Elivar, E. Belmont, E. Benítez, S. Y. BenZvi, D. Berley, A. Bernal, E. Bonamente, J. Braun, R. Caballero-Lopez, I. Cabrera, A. Carramiñana, L. Carrasco, M. Castillo, L. Chambers, R. Conde, P. Condreay, U. Cotti, J. Cotzomi, J. C. D'Olivo, E. de la Fuente, C. De León, S. Delay, D. Delepine, T. DeYoung, L. Diaz, L. DiazCruz, B. L. Dingus, M. A. Duvernois, D. Edmunds, R. W. Ellsworth, B. Fick, D. W. Fiorino, A. Flandes, N. I. Fraija, A. Galindo, J. L. Garcia-Luna, G. Garcia-Torales, F. Garfias, L. X. González, M. M. González, J. A. Goodman, V. Grabski, M. Gussert, C. GuzmánCeron, Z. Hampel-Arias, T. Harris, E. Hays, L. Hernandez-Cervantes, P. H. Hüntemeyer, A. Imran, A. Iriarte, J. J. Jimenez, P. Karn, N. Kelley-Hoskins, D. Kieda, R. Langarica, A. Lara, R. Lauer, W. H. 
Lee, E. C. Linares, J. T. Linnemann, M. Longo, R. Luna-García, H. Martınez, J. Martínez, L. A. Martınez, O. Martınez, J. MartınezCastro, M. Martos, J. Matthews, J. E. McEnery, G. Medina-Tanco, J. E. Mendoza-Torres, P. A. Miranda-Romagnoli, T. Montaruli, E. Moreno, M. Mostafa, M. Napsuciale, J. Nava, L. Nellen, M. Newbold, R. Noriega-Papaqui, T. Oceguera-Becerra, A. Olmos Tapia, V. Orozco, V. Pérez, E. G. Pérez-Pérez, J. S. Perkins, J. Pretz, C. Ramirez, I. Ramírez, D. Rebello, A. Rentería, J. Reyes, D. Rosa-González, A. Rosado, J. M. Ryan, J. R. Sacahui, H. Salazar, F. Salesa, A. Sandoval, E. Santos, M. Schneider, A. Shoup, S. Silich, G. Sinnis, A. J. Smith, K. Sparks, W. Springer, F. Suárez, N. Suarez, I. Taboada, A. F. Tellez, G. Tenorio-Tagle, A. Tepe, P. A. Toale, K. Tollefson, I. Torres, T. N. Ukwatta, J. Valdes-Galicia, P. Vanegas, V. Vasileiou, O. Vázquez, X. Vázquez, L. Villaseñor, W. Wall, J. S. Walters, D. Warner, S. Westerhoff, I. G. Wisher, J. Wood, G. B. Yodh, D. Zaborov, and A. Zepeda, "On the sensitivity of the HAWC observatory to gamma-ray bursts," Astroparticle Physics, vol. 35, pp. 641-650, May 2012.

[15] J. H. MacGibbon, "Quark- and gluon-jet emission from primordial black holes. II. The emission over the black-hole lifetime," Phys. Rev. D, vol. 44, pp. 376-392, July 1991.

[16] V. B. Petkov, E. V. Bugaev, P. A. Klimai, M. V. Andreev, V. I. Volchenko, G. V. Volchenko, A. N. Gaponenko, Z. S. Guliev, I. M. Dzaparova, D. V. Smirnov, A. V. Sergeev, A. B. Chernyaev, and A. F. Yanin, "Searching for very-high-energy gamma-ray bursts from evaporating primordial black holes," Astronomy Letters, vol. 34, pp. 509-514, Aug. 2008.

[17] V. Vasileiou, A search for bursts of very high energy gamma rays with Milagro. PhD thesis, University of Maryland, College Park, 2008.

[18] A. A. Abdo, B. T. Allen, R. Atkins, T. Aune, W. Benbow, D. Berley, E. Blaufuss, E. Bonamente, J. Bussons, C. Chen, G. E. Christopher, D. G. Coyne, T. DeYoung, B. L. Dingus, D. E. Dorfan, R. W. Ellsworth, A. Falcone, L. Fleysher, R. Fleysher, J. Galbraith-Frew, M. M. Gonzalez, J. A. Goodman, T. J. Haines, E. Hays, C. M. Hoffman, P. H. Hüntemeyer, L. A. Kelley, B. E. Kolterman, C. P. Lansdell, J. T. Linnemann, J. McCullough, J. E. McEnery, T. Morgan, A. I. Mincer, M. F. Morales, P. Nemethy, D. Noyes, J. Pretz, J. M. Ryan, F. W. Samuelson, P. M. Saz Parkinson, A. Shoup, G. Sinnis, A. J. Smith, G. W. Sullivan, 
V. Vasileiou, G. P. Walker, M. Wascko, D. A. Williams, S. Westerhoff, and G. B. Yodh, "Observation and Spectral Measurements of the Crab Nebula with Milagro," ApJ., vol. 750, p. 63, May 2012.

[19] A. D. Panov, J. H. Adams, H. S. Ahn, G. L. Bashindzhagyan, K. E. Batkov, J. Chang, M. Christl, A. R. Fazely, O. Ganel, R. M. Gunashingha, T. G. Guzik, J. Isbert, K. C. Kim, E. N. Kouznetsov, M. I. Panasyuk, W. K. H. Schmidt, E. S. Seo, N. V. Sokolskaya, J. W. Watts, J. P. Wefel, J. Wu, and V. I. Zatsepin, "The results of ATIC-2 experiment for elemental spectra of cosmic rays," ArXiv Astrophysics e-prints, Dec. 2006.

[20] M. Amenomori, Z. Cao, B. Z. Dai, L. K. Ding, Y. X. Feng, Z. Y. Feng, K. Hibino, N. Hotta, Q. Huang, A. X. Huo, H. Y. Jia, G. Z. Jiang, S. Q. Jiao, F. Kajino, K. Kasahara, Y. Kitahara, Labaciren, S. M. Liu, D. M. Mei, L. Meng, X. R. Meng, Mimaciren, K. Mizutani, J. Mu, H. Nanjo, M. Nishizawa, A. Oguro, M. Ohnishi, I. Ohta, T. Ouchi, J. R. Ren, T. Saito, M. Sakata, Z. Z. Shi, M. Shibata, T. Shirai, H. Sugimoto, X. X. Sun, K. Taira, Y. H. Tan, N. Tateyama, S. Torii, H. Wang, C. Z. Wen, Y. Yamamoto, G. C. Yu, P. Yuan, C. S. Zhang, H. M. Zhang, L. Zhang, Zhasang, Zhaxiciren, and W. D. Zhou, "Search for $10 \mathrm{TeV}$ Gamma Bursts from Evaporating Primordial Black Holes with the Tibet Air Shower Array," International Cosmic Ray Conference, vol. 2, p. 112, 1995 .

[21] D. E. Alexandreas, G. E. Allen, D. Berley, S. Biller, R. L. Burman, M. Cavalli-Sforza, C. Y. Chang, M. L. Chen, P. Chumney, D. Coyne, C. Dion, G. M. Dion, D. Dorfan, R. W. Ellsworth, J. A. Goodman, T. J. Haines, M. Harmon, C. M. Hoffman, L. Kelley, S. Klein, D. E. Nagle, D. M. Schmidt, R. Schnee, C. Sinnis, A. Shoup, M. J. Stark, D. D. Weeks, D. A. Williams, J. P. Wu, T. Yang, G. B. Yodh, and W. P. Zhang, "New limit on the rate-density of evaporating black holes," Physical Review Letters, vol. 71, pp. 2524-2527, Oct. 1993.

[22] E. T. Linton, R. W. Atkins, H. M. Badran, G. Blaylock, P. J. Boyle, J. H. Buckley, K. L. Byrum, D. A. Carter-Lewis, O. Celik, Y. C. K. Chow, P. Cogan, M. K. Daniel, C. Dowdall, A. D. Falcone, D. J. Fegan, S. J. Fegan, J. P. Finley, P. Fortin, K. J. Guiterrez, J. Hall, D. Hanna, J. Holder, D. Horan, S. B. Hughes, T. B. Humensky, I. Jung, G. E. Kenny, M. Kertzman, D. B. Kieda, J. Kildea, J. Knapp, H. Krawczynski, M. J. Lang, S. LeBohec, G. Maier, P. Moriarty, R. A. Ong, J. S. 
Perkins, F. Pizlo, M. Pohl, J. Quinn, K. Ragan, P. F. Rebillot, P. T. Reynolds, G. H. Sembroski, D. Steele, S. P. Swordy, L. Valcarcel, S. P. Wakely, T. C. Weekes, and R. J. White, "A new search for primordial black hole evaporations using the Whipple gamma-ray telescope," JCAP, vol. 1, p. 13, Jan. 2006.

[23] G. Tešić and VERITAS Collaboration, "Searching for primordial black holes with the VERITAS gamma-ray experiment," Journal of Physics Conference Series, vol. 375, p. 052024, July 2012.

[24] B. J. Carr, "Some cosmological consequences of primordial black-hole evaporations," ApJ., vol. 206, pp. 8-25, May 1976.

[25] J. Holmberg and C. Flynn, "The local surface density of disc matter mapped by Hipparcos," MNRAS, vol. 352, pp. 440-446, Aug. 2004.

[26] J. Bovy and S. Tremaine, "On the Local Dark Matter Density," ApJ., vol. 756, p. 89, Sept. 2012.

[27] B. J. Carr and J. H. MacGibbon, "Cosmic rays from primordial black holes and constraints on the early universe.," Phy. Rep., vol. 307, pp. 141-154, Dec. 1998.

[28] J. H. MacGibbon and B. J. Carr, "Cosmic rays from primordial black holes," ApJ., vol. 371, pp. 447-469, Apr. 1991. 